\title{
Reconstruction method incorporating the object-position dependence of visibility loss in dark-field imaging
}

\author{
Udo van Stevendaal $^{1}$, Zhentian Wang $^{2}$, Thomas Köhler ${ }^{1}$, \\ Gerhard Martens ${ }^{1}$, Marco Stampanoni ${ }^{2,3}$, Ewald Roess ${ }^{1}$ \\ ${ }^{1}$ Philips Research Laboratories, Innovative Technologies, Hamburg, Germany, \\ ${ }^{2}$ Swiss Light Source, Paul Scherrer Institut, 5232 Villigen, Switzerland \\ ${ }^{3}$ Institute for Biomedical Engineering, University and ETH Zürich, 8092 Zürich, Switzerland
}

\begin{abstract}
Dark-field imaging has the potential to overcome limitations in computed tomography (CT) investigating relatively weakly absorbing material. However, an object-position dependence of the visibility loss in dark-field imaging is observed. This effect might be negligible for small objects, but, for acquisition geometries using fanangle apertures and field of views as those in human CT scanners, the object-position dependence of visibility loss has to be taken into consideration if the scattering structure within the object is in the range of the grating periods, i.e. micrometer. This work examines the effect of object-position dependent visibility loss in dark-field imaging experimentally, investigates its consequences and presents an algorithm which solves the corresponding reconstruction problem.
\end{abstract}

Keywords: Differential Phase-Contrast Imaging, Dark-Field Imaging, Phase-Contrast CT

\section{INTRODUCTION}

Differential phase-contrast imaging (PCI) in the x-ray domain provides three physically complementary signals ${ }^{1,2}$ : the attenuation, the differential phase-contrast - related to the refractive index - and the dark-field signal - related to the total amount of radiation scattered into very small angles. In cases where different forms of tissue with similar absorption cross-sections are under investigation, e.g. mammography or angiography, the x-ray absorption contrast is relatively poor. Consequently, differentiating pathologic from non-pathologic tissue in an absorption radiograph obtained with a current hospital-based x-ray system remains practically impossible for many tissue compositions. Dark-field imaging has the potential to overcome the above-noted contrast limitation. ${ }^{3,4}$ One particular problem with dark-field imaging is that the signal creation process is quite complex (see Refs. 5,6) and depends on the direction of the sample relative to the gratings. ${ }^{7,8}$ Another complication arises from one feature that has been mentioned parenthetically by Yashiro et al. ${ }^{6}$ namely that the signal also depends on the position of the sample along the ray. To the best of our knowledge, this theoretically predicted feature has not been described experimentally. We will present experimental results on this observation in Sec. 2.1. A detailed experimental and analytical investigation of this effect is work in progress and shall be published elsewhere.

Of course, there is a desire to use the dark-field signal for tomographic reconstruction. However, there are currently only algorithms available, which make substantial simplifications in the description of the signal creation process: We are only aware of the use of the conventional filtered back-projection (FBP) approach for reconstructing dark-field images in computed tomography. ${ }^{3,9,10}$ This approach explicitly ignores the dependence of the direction of the sample and is therefore valid only for isotropic materials. The approach also ignores objectdependence of the visibility loss along the beam, presumably because the authors were not aware of this not yet widely known feature. This second simplification limits the method further to situations where the parallel beam approximation holds, i.e., to cases with rather small fan angle. In this contribution, we demonstrate the influence of the object position in dark-field images if the scattering structure within the object is in the range of the grating periods, i.e. micrometer, by performing measurements (Sec. 2.1) as well as CT like simulations. Furthermore, we present two different algorithms which solve the reconstruction problem (Sec. 2.2) and apply these algorithms to simulated data (Sec. 3).

Send correspondence to Udo van Stevendaal, E-mail: udo.van.stevendaal@philips.com

Medical Imaging 2013: Physics of Medical Imaging, edited by Robert M. Nishikawa, Bruce R. Whiting, Christoph Hoeschen, Proc. of SPIE Vol. 8668, 86680Z · (c) 2013 SPIE · CCC code: 1605-7422/13/\$18 · doi: 10.1117/12.2006711 


\section{METHOD}

Most promising for medical applications is the grating-based phase-contrast imaging technique because of its use of conventional x-ray tubes and x-ray detectors (see Ref. 1). Three gratings are needed to generate the signal information. The first grating $\left(\mathrm{G}_{0}\right)$ is an absorption grating and generates partially coherent $\mathrm{x}$-rays, enabling the observation of interference phenomena. The second, phase grating $\left(\mathrm{G}_{1}\right)$ and the third, absorption grating $\left(\mathrm{G}_{2}\right)$ form the interferometer unit which is needed to analyze the wavefront modified by an x-rayed object. In order to sample the interference pattern with structure sizes around one micrometer, too small for conventional detector pixels to resolve, either $\mathrm{G}_{1}$ or $\mathrm{G}_{2}$ is translated in numerous steps over a multiple of the grating period. In Fig. 1 the signal sampling is exemplarily shown for translating the absorption grating $G_{2}$ in eight steps over one grating period $p_{2}$. From two acquisitions with and without an object, the three physically complementary

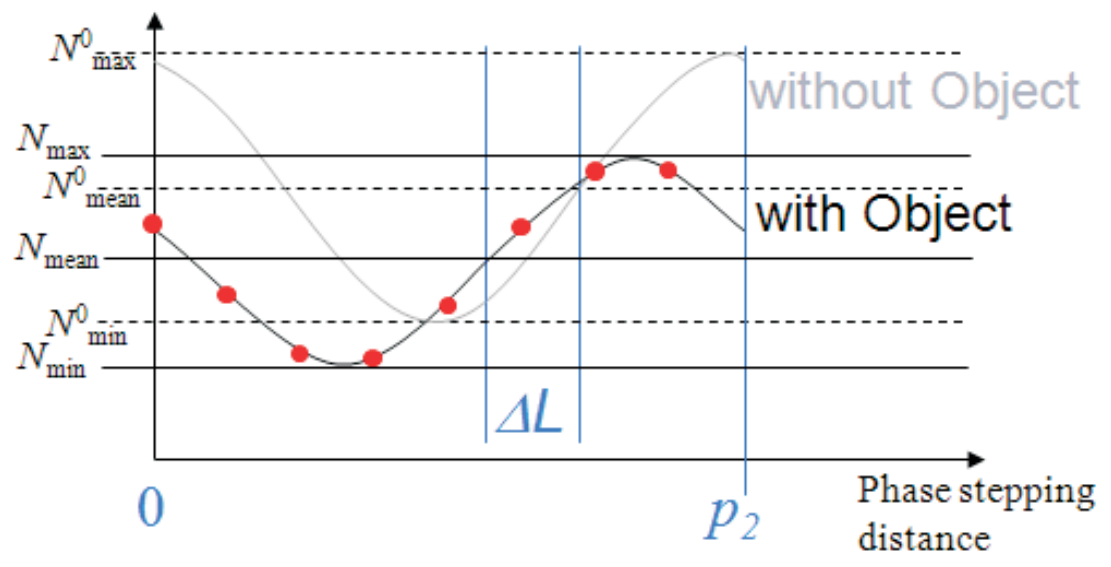

Figure 1. Exemplary signal retrieval of a phase-contrast image acquisition.

signals, the attenuation, the differential phase-contrast, and the dark field can be deduced as follows: 1.) The absorption image $i m g_{\mu}$ is given by the mean signal intensity to $i m g_{\mu}=-\ln \left(I / I_{0}\right)$, with $\left(I=N_{\text {mean }}\right)$ and without $\left(I_{0}=N_{\text {mean }}^{0}\right)$ the object. 2.) Essential for the differential phase-contrast image is the phase shift $\Delta L$ of the two sinusoidal curves obtained with and without the object. From this quantity the differential of line integrals of the refractive index of the object can be retrieved by a one-dimensional integration according to Ref. 11. 3.) If the background visibility $V_{0}$ is given by $V_{0}=\left(N_{\max }^{0}-N_{\min }^{0}\right) /\left(N_{\max }^{0}+N_{\min }^{0}\right)$, the dark-field image $\left(i m g_{\sigma}\right)$ or the normalized visibility loss is calculated by $i m g_{\sigma}=-\ln \left(V / V_{0}\right)$, where $V=\left(N_{\max }-N_{\min }\right) /\left(N_{\max }+N_{\min }\right)$ is the visibility in the presence of the object. As an exemplary result of a phase-contrast image acquisition Fig. 2 shows the absorption, the dark-field, and the differential-phase contrast images of a mouse. Because it offers the opportunity to overcome the intrinsically weak soft-tissue absorption contrast of x-ray imaging modalities, phase-contrast and especially dark-field imaging are the subject of active current research. However, there might be limitations which is shown in the following.

\subsection{Experiment: Visibility loss in dark-field imaging}

Our PCI setup consists of a conventional mammography x-ray tube (C340 V, $500 \mathrm{~W}$, I.A.E Spa - Italy) and a Shad-o-Box ${ }^{\mathrm{TM}} 2048$ detector (1024 x 2048 pixel array with $48 \mu \mathrm{m}$ pixel spacing, Rad-icon Imaging Corp, USA). The distances between the gratings $\mathrm{G}_{0}$ and $\mathrm{G}_{1}$ are $825 \mathrm{~mm}$ and between $\mathrm{G}_{1}$ and $\mathrm{G}_{2}$ are $82.5 \mathrm{~mm}$, respectively (see Figs. 3 and 4). Twenty five layers of commercial post-it paper stripes are stuck together and are placed right in front the phase grating $\mathrm{G}_{1}$. A electron microscope recording revealed fiber structures with diameters in the micrometer range. A data acquisition is performed with a voltage of $37 \mathrm{kV}$ and a current of $120 \mathrm{~mA}$, respectively. While the phase grating is translated in 16 steps over one grating period, i.e. $0.338 \mu \mathrm{m}$ per step, the object is x-rayed for $500 \mathrm{~ms}$ after each step. A second acquisition is performed in the same way without the object and the normalized visibility loss is calculated according to $i m g_{\sigma}=-\ln \left(V / V_{0}\right)$. This procedure is repeated for increasing object distances with respect to the phase grating $\mathrm{G}_{1}$ (see Fig. 4). 


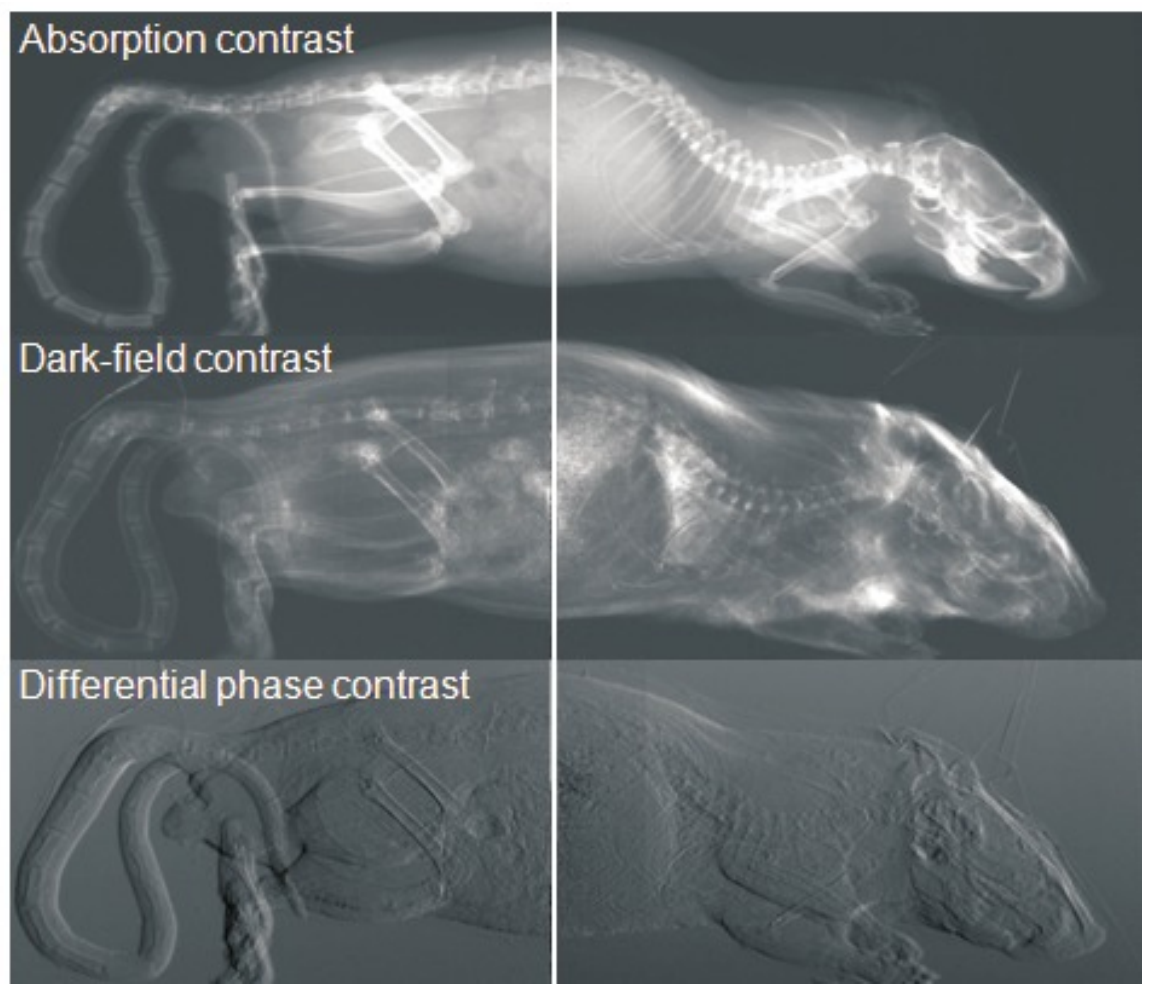

Figure 2. Absorption, dark-field, and differential-phase contrast images of a mouse.
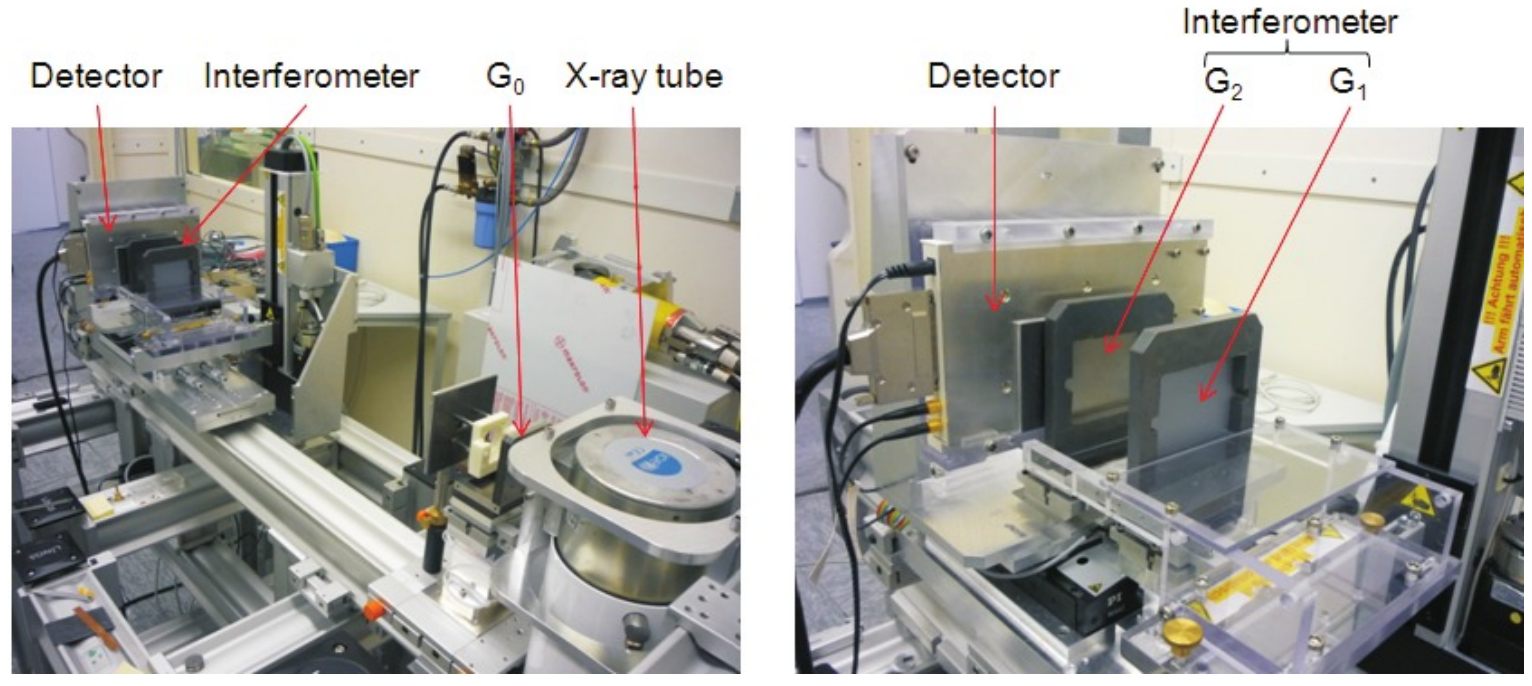

Figure 3. PCI lab setup.

It can be seen in Fig. 5 that the visibility loss decreases with an increasing object distance from $\mathrm{G}_{1}$ in the direction to $\mathrm{G}_{0}$. Taking the $x$-axis interception $A=0.8715$ and the slope $B=-0.011$, the interception of the regression line with the $x$-axis, i.e. $y=0$, is calculated to $x(y=0)=|A / B|=79.2 \pm 14.7$, where the error is given by a standard error propagation. ${ }^{12}$ From this, it can be derived that the visibility loss should be zero in front of $\mathrm{G}_{0}$. Within the measurement errors, this is in good agreement with the magnification $\mathcal{M}$ of the setup 


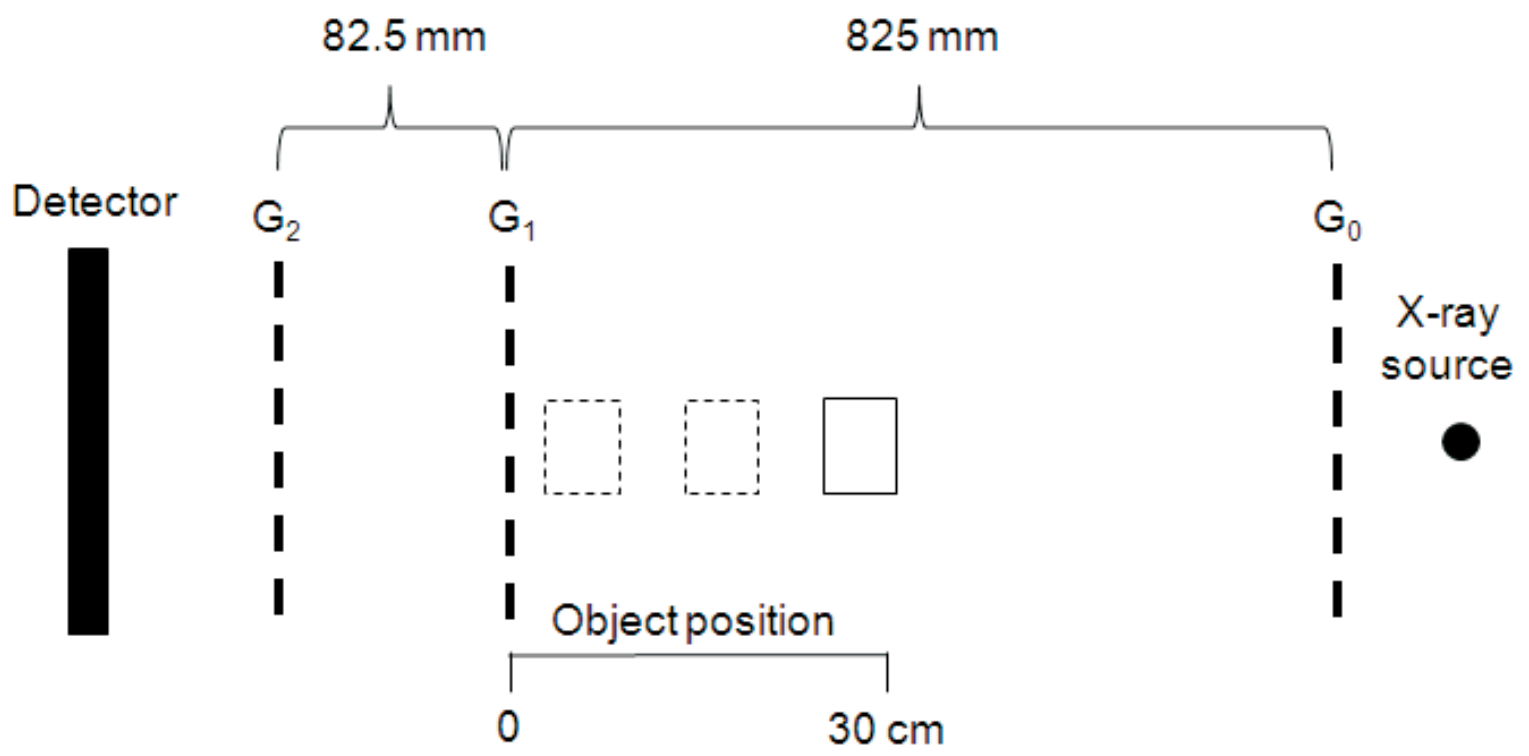

Figure 4. Sketch of the experimental arrangement.

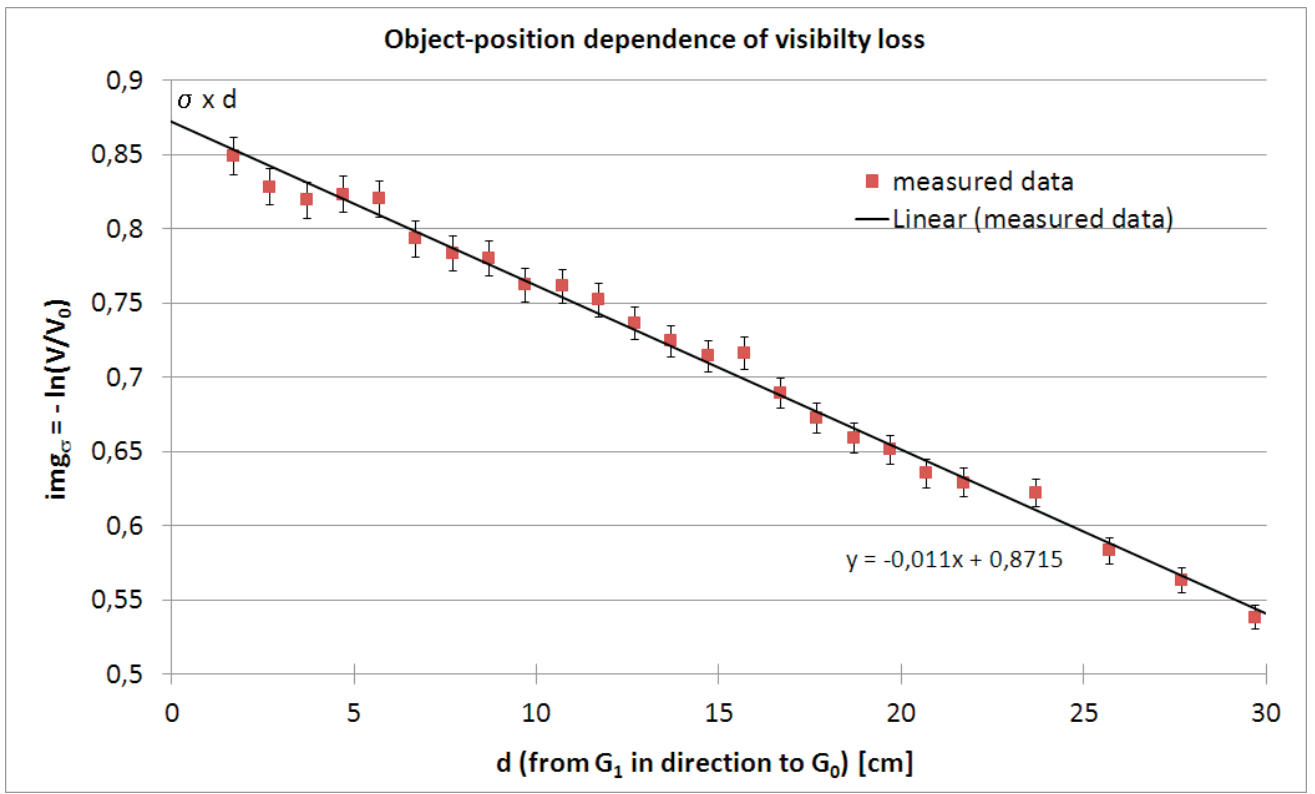

Figure 5. Visibility loss as a function of the distance of the object from $\mathrm{G}_{1}$ towards $\mathrm{G}_{0}$.

according to

$$
\mathcal{M}=\frac{82.5 \mathrm{~cm}}{82.5 \mathrm{~cm}-d_{\text {object }}},
$$

where $d_{\text {object }}$ is the distance of the object from $\mathrm{G}_{1}$ towards $\mathrm{G}_{0}$. It is worth mentioning that the geometry of the experimental setup produces a fan-beam of x-rays.

If dark-field imaging is applied in $\mathrm{CT}$, the effect of an object-position dependent visibility loss might be negligible for small objects. However, acquisition geometries using fan-angle apertures and field of views (FOV) as those in human CT scanners might suffer from the object-position dependence of visibility loss. This is investigated in more detail in the following. 


\subsection{Theory}

First, we review briefly the basics of absorption contrast imaging: For the attenuation of the x-ray along a path the resulting intensity $I$ follows Lambert-Beer's-Law (see also Fig. 6, left):

$$
I=I_{0} \exp \left(-\int_{0}^{L} \mu(\vec{s}+l \vec{n}) \mathrm{d} l\right),
$$

where $I_{0}$ is the incoming intensity, $L$ is the length of the x-ray path from the source through the object to the detector pixel, $\mu$ the linear attenuation coefficient, $\vec{s}$ the source position and $\vec{n}$ a unit vector along the x-ray beam direction. Dividing by $I_{0}$ and taking the logarithm of both sides of the equation leads to a linear equation
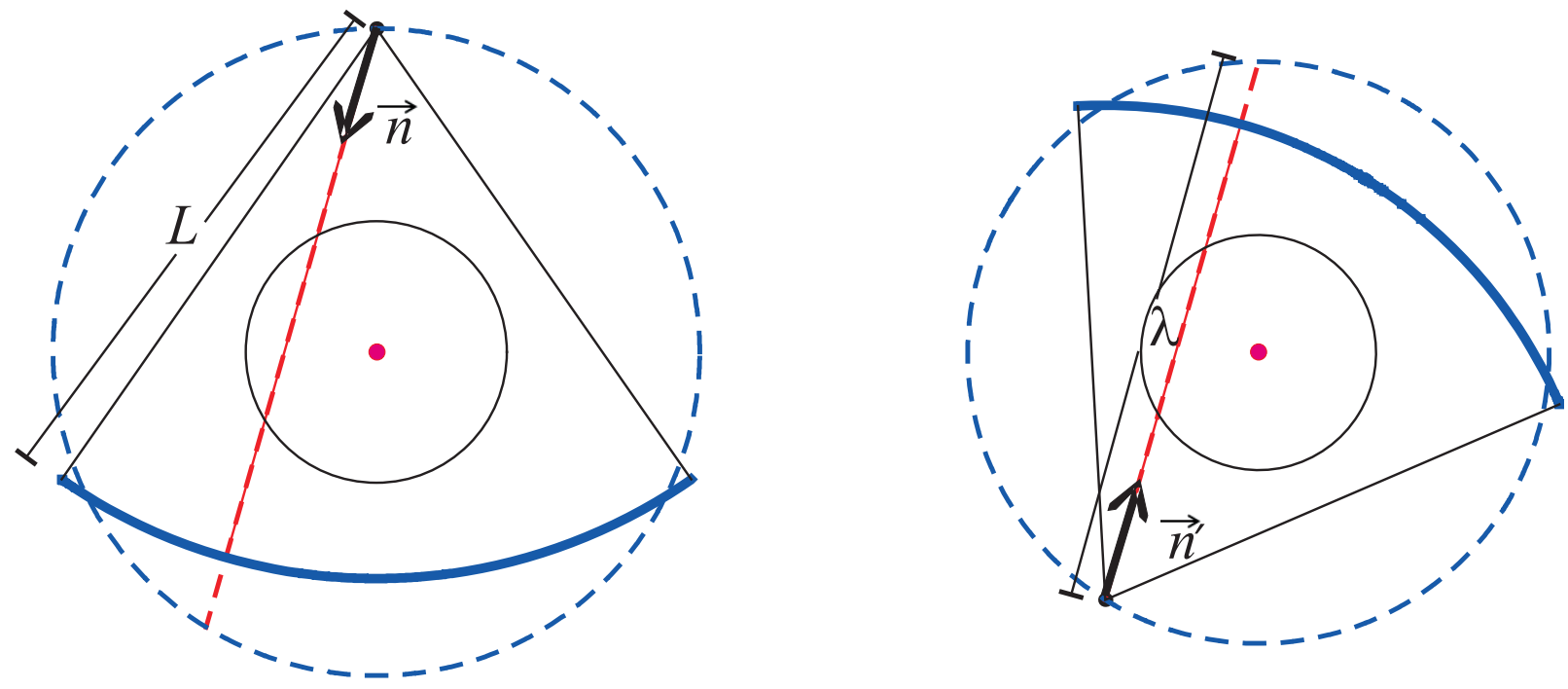

Figure 6. Sketch of a focus-centered CT geometry to explain the occurrence of complementary rays.

representing the line integral of the attenuation coefficient along the path:

$$
i m g_{\mu}=-\ln \left(I / I_{0}\right)=\int_{0}^{L} \mu(\vec{s}+l \vec{n}) \mathrm{d} l,
$$

where $i m g_{\mu}$ is independent with respect to the displacement of the object along $\vec{n}$.

With dark-field imaging, the respective measured projections are scaled depending on the object position similar to attenuation contrast imaging, but the values of line integrals scale inversely with the distance from the source if the scattering structure within the object is in the range of the grating periods, i.e. typically micrometer. For dark-field imaging of such object details, this leads to a loss of visibility $V$ along a path according to:

$$
V=V_{0} \exp \left(-\int_{0}^{L} \frac{l}{L} \sigma(\vec{s}+l \vec{n}) \mathrm{d} l\right) .
$$

The material property $\sigma$ denotes the scatter coefficient. In the following, we restrict ourselves to the case where the scattering is independent of the incident direction of the x-ray beam. In analogy to the attenuation coefficient but rather to a weighted line integral the following equation is given:

$$
i m g_{\sigma}=-\ln \left(V / V_{0}\right)=\int_{0}^{L} \frac{l}{L} \sigma(\vec{s}+l \vec{n}) \mathrm{d} l .
$$

The aim of dark-field computed tomography is to reconstruct the spatial distribution of the scatter coefficient $\sigma$ from a set of measurements of the dark-field signal $i m g_{\sigma}$. 
The factor $l / L$ can be taken into account correctly into the reconstruction process straightforwardly if iterative reconstruction algorithms are used: Since the forward problem is linear even with the additional factor, any iterative reconstruction algorithm that solves a linear system, e.g. ART or SART, can still be used without modification to the algorithm - except of course that the correct forward model is used. Here, we will use an SART as an iterative reconstruction algorithm.

The situation is more complicated for filtered back-projection. The object-position dependence of visibility loss is not taken into account in conventional fan-beam FBP algorithms and the additional weighting $l / L$ does not permit to use such algorithms to reconstruct the distribution of the scatter coefficient. Only in case the weighting factor changes little over the object's extend, for example if the object is very small compared to the distance $L$, the additional weighting can be approximated to be constant or dropped.

In the following, we derive a new FBP type algorithm that overcomes this limitation. The basic idea is to use information from complementary rays, i.e., rays, which are geometrically the same, but taken at opposite directions (see Fig. 6). In conventional CT, these rays are truly equivalent. However, in the case of dark-field imaging with the additional weighting, complementary rays no longer give necessarily identical results. Starting with:

$$
V^{\prime}=V_{0}^{\prime} \exp \left(-\int_{0}^{L^{\prime}} \frac{l}{L^{\prime}} \sigma\left(\vec{s}^{\prime}+l \vec{n}^{\prime}\right) \mathrm{d} l\right),
$$

where the primes indicate that the geometrical values relate to the complementary x-ray, we obtain

$$
i m g_{\sigma}^{\prime}=-\ln \left(V^{\prime} / V_{0}^{\prime}\right)=\int_{0}^{L^{\prime}} \frac{l}{L^{\prime}} \sigma\left(\vec{s}^{\prime}+l \vec{n}^{\prime}\right) \mathrm{d} l=\int_{0}^{L} \frac{l}{L} \sigma\left(\vec{s}^{\prime}-l \vec{n}\right) \mathrm{d} l
$$

exploiting that by definition of the complementary ray, the relation $\vec{n}^{\prime}=-\vec{n}$ holds true. Furthermore, $L=L^{\prime}$ is assumed, e.g. for circular trajectories.

The source position for the complementary x-ray is somewhere on the ray $\vec{s}=\vec{s}+\lambda \vec{n}$ for some $\lambda$. Substituting this into Eq. (7), changing the variables $l^{\prime}=\lambda-l$ and assuming that the scatter coefficient distribution outside the field of view is zero yields:

$$
i m g_{\sigma}^{\prime}=\frac{1}{L} \int_{0}^{L}\left(\lambda-l^{\prime}\right) \sigma\left(\vec{s}+l^{\prime} \vec{n}\right) \mathrm{d} l^{\prime} .
$$

Summing $i m g_{\sigma}$ and $i m g_{\sigma}^{\prime}$ to

$$
i m g_{\sigma}+i m g_{\sigma}^{\prime}=\frac{\lambda}{L} \int_{0}^{L} \sigma(\vec{s}+l \vec{n}) \mathrm{d} l
$$

leads to the following equation which can be solved by using a conventional FBP algorithm to reconstruct the scatter coefficient distribution:

$$
\frac{L}{\lambda}\left(i m g_{\sigma}+i m g_{\sigma}^{\prime}\right)=\int_{0}^{L} \sigma(\vec{s}+l \vec{n}) \mathrm{d} l
$$

In practice, the dark-field signal will contain contributions from object features that scale with the position and contributions which do not. In this publication, we restrict ourself to the case of objects with scattering structures in the micrometer range.

In general, the weighting factor $L / \lambda$ depends on the fan angle. We further note that there is one particular geometry, where this factor is constant, namely a geometry where the detector follows exactly the source path. This geometry is illustrated in Fig. 7. Using this geometry would permit to take into account correctly the signal contribution which scale with the position and which do not. This is due to the fact that Eq. (10) boils down to a simple average, which is correct for both signal types. Unfortunately, the dedicated geometry has other disadvantages like inefficient usage of detector area and thus, it is unlikely being used.

Note that the FBP-type algorithm outlined here can be easily extended to helical acquisition if 360 degree interpolation is employed. However, it cannot be extended to cone-beam acquisitions since only very few rays are measured in a truly redundant manner. 


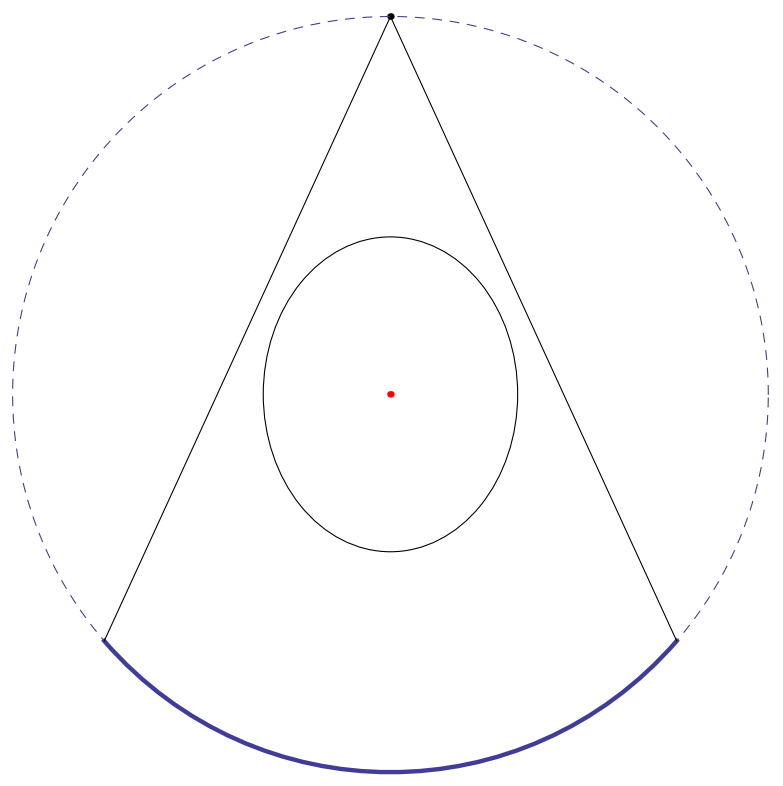

Figure 7. Sketch of CT geometry where the detector follows exactly the source path.

\section{NUMERICAL SIMULATIONS AND RESULTS}

The influence of the effect being investigated here on tomographic reconstruction is studied in simulations. The simulation was performed using a discrete blob-driven forward projection of a discrete version of the forbild head phantom $(512 \times 512)$. In addition to the standard blob-driven forward projection, each footprint was scaled with $l / L$ according to Eq. (5). The effect of varying scaling within the blob was neglected since the blob-extend is more than two orders of magnitude smaller than the phantom. First, Fig. 8 illustrates, how a sinogram changes if the position-dependent scaling is taken into account during forward projection. The lower left figure shows the

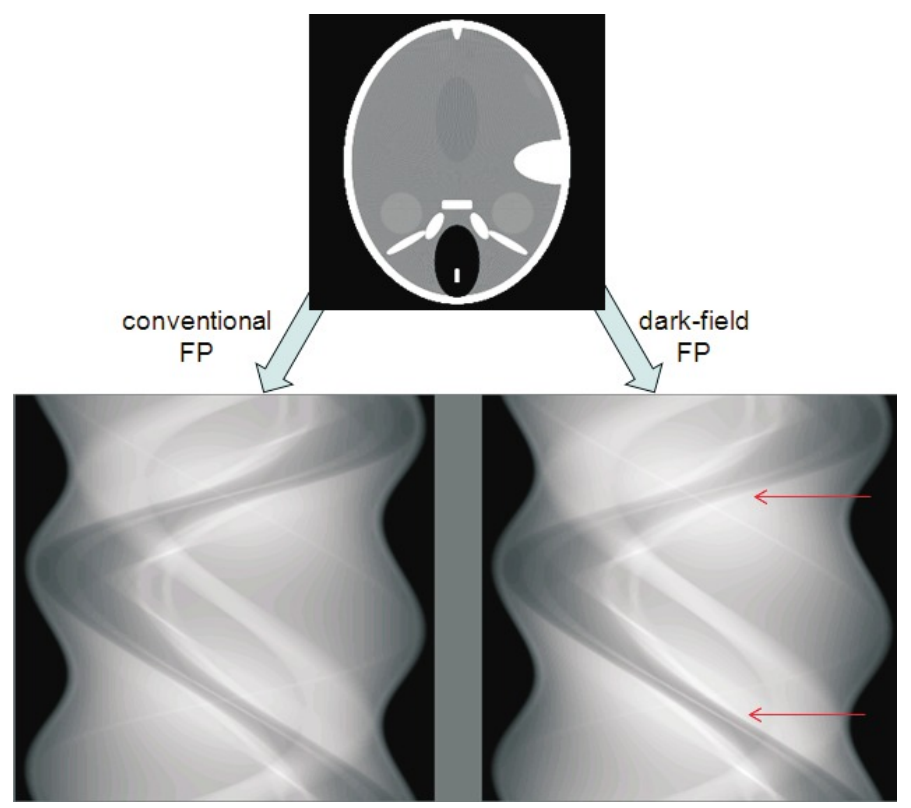

Figure 8. Sinograms for the simulation of the forbild head phantom in conventional (left image) and dark-field mode (right image), where the position-dependent scaling is included. 
sinogram for a conventional forward projection (conventional FP) while the lower right figure depicts a sinogram for the dark-field forward projection (dark-field FP). Due to the position-dependent scaling, the contrast in the projection data is changed, most prominently visible for the frontal sinus (air cavity at the bottom of the phantom), which creates a dark sinusoidal trace in the sinogram. When the source is close to the cavity, its contrast is decreased (upper arrow) whereas it is increased if the source is far away from it (lower arrow).

Next, we study how the use of conventional FBP that ignores the position-dependent scaling influences image quality. The aim here is to show what kind of artifacts are expected if the position-dependent scaling is ignored during reconstruction. Fig. 9 illustrates that a standard filtered back-projection is not appropriate for the darkfield projection data (right image). In addition, the FBP reconstruction of conventional forward projected data is shown in the left image. For a fair comparison, the same settings for level and window, given in Hounsfield units (HU), are chosen to visualize both images with a dedicated viewer. These values are mentioned in the figure description even if the images do not represent real Hounsfield units. This holds true for all images in the following.

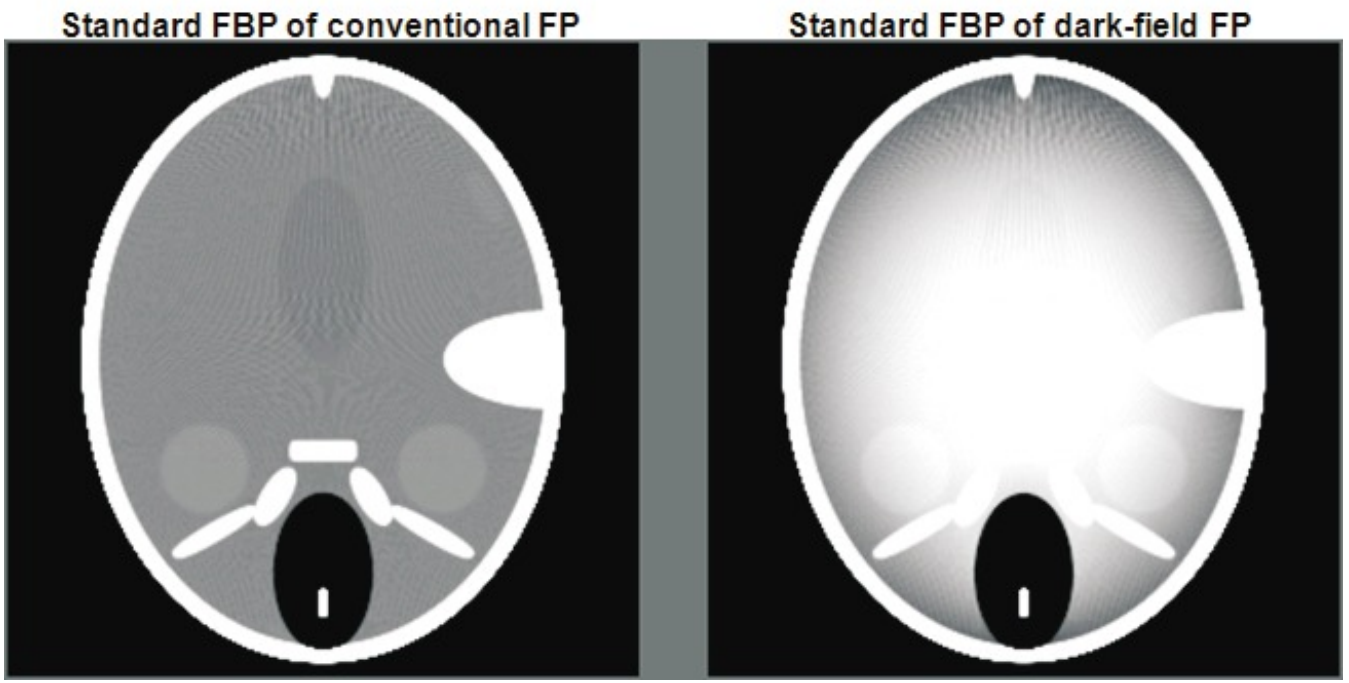

Figure 9. Filtered back-projection image results of the forbild head phantom. Left: Conventional forward projected data are taken into account. Right: Dark-field forward projected data are considered (see also Fig. 8). Level and window are 50 and $200 \mathrm{HU}$, respectively.

The same comparison as in Fig. 9 is done in Fig. 10 for an SART reconstruction algorithm. Ten iterations with seven projections per subset were used. If the position-dependent scaling is ignored, the main structure of the artifact, namely a capping, is the same as in FBP. Of course, we expect that image quality degrades with increasing fan angle. Therefore, we simulated scanners with constant field of view, but different fan angles. Results of this simulation are shown in Fig. 11. The upper row of the reconstructed images shows the result if the reconstruction of the dark-field signal is performed with a conventional FBP algorithm. Strong capping artifacts occur which are reduced at a smaller fan. The second row of FBP reconstructed absorption images shall demonstrate that there is no comparable effect for the attenuation signal. If the object-position dependent visibility loss is considered in the reconstruction algorithm, for instance by a proper SART, the dark-field signal is correctly taken into account and the capping vanishes (see lower row of reconstructed images in Fig. 11).

Similar simulations are also performed regarding the object position within the field of view (see Fig. 12). Again the upper row shows the reconstruction results of the dark-field signal with a conventional FBP algorithm. These images deploy a signal drop dependent on the object position. For comparison, the second row contains the FBP reconstructed absorption images. A correct SART of the dark-field signal leads to the images of the lower row in Fig. 12. 


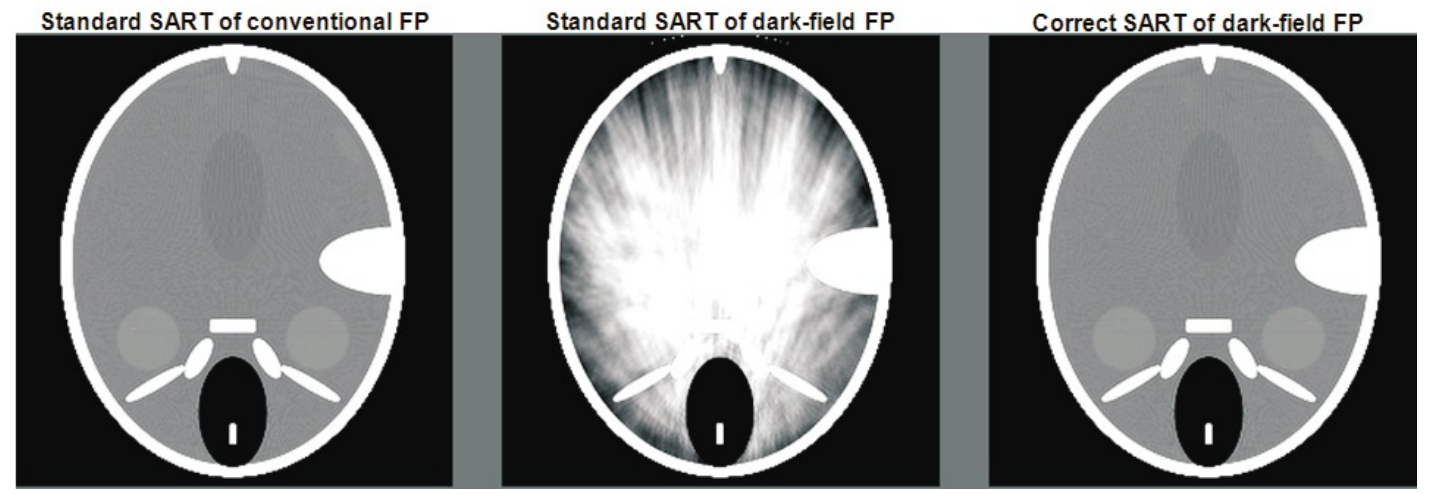

Figure 10. Comparison of the reconstruction results for the forbild head phantom obtained with standard SART for the conventional mode (left image), with standard SART for the dark-field mode (middle image), and with correct SART for the dark-field mode (right image) Level and window are 50 and 200 HU, respectively.
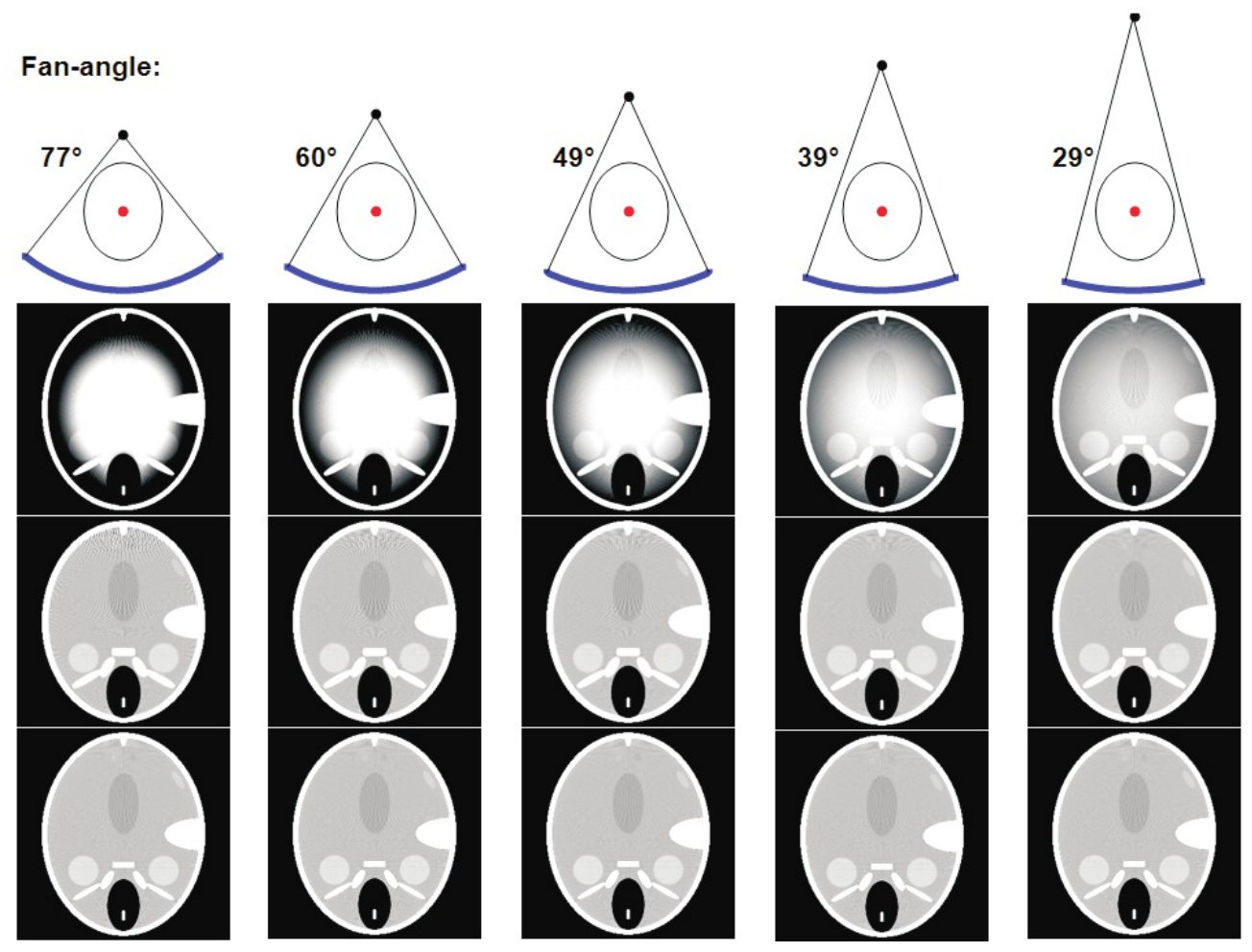

Figure 11. Effect of object-position dependence of visibility loss for various scanner geometries, i.e. fan angles. The upper and middle row of the reconstructed images show the result of a standard FBP reconstruction for the dark-field and attenuation images, respectively. The lower row shows the result of SART for the dark-field images. Level (35 HU) and window $(70 \mathrm{HU})$ are the same for all images.

\section{DISCUSSION}

If the scattering structure within the object is in the range of the grating periods, i.e. micrometer, it is found that the visibility loss depends on the object position. This can be observed in Fig. 5, where paper stripes were used as object. The visibility loss scales well in line with the model as given in Eq. (5). We verified that this effect has an influence on phase-contrast CT using simulations. Fig. 10 shows a dark-field SART reconstruction of simulated data of the forbild head phantom without (middle) and with (right) considering the influence of the object position in the reconstruction algorithm. It can be observed that a conventional reconstruction leads to capping 


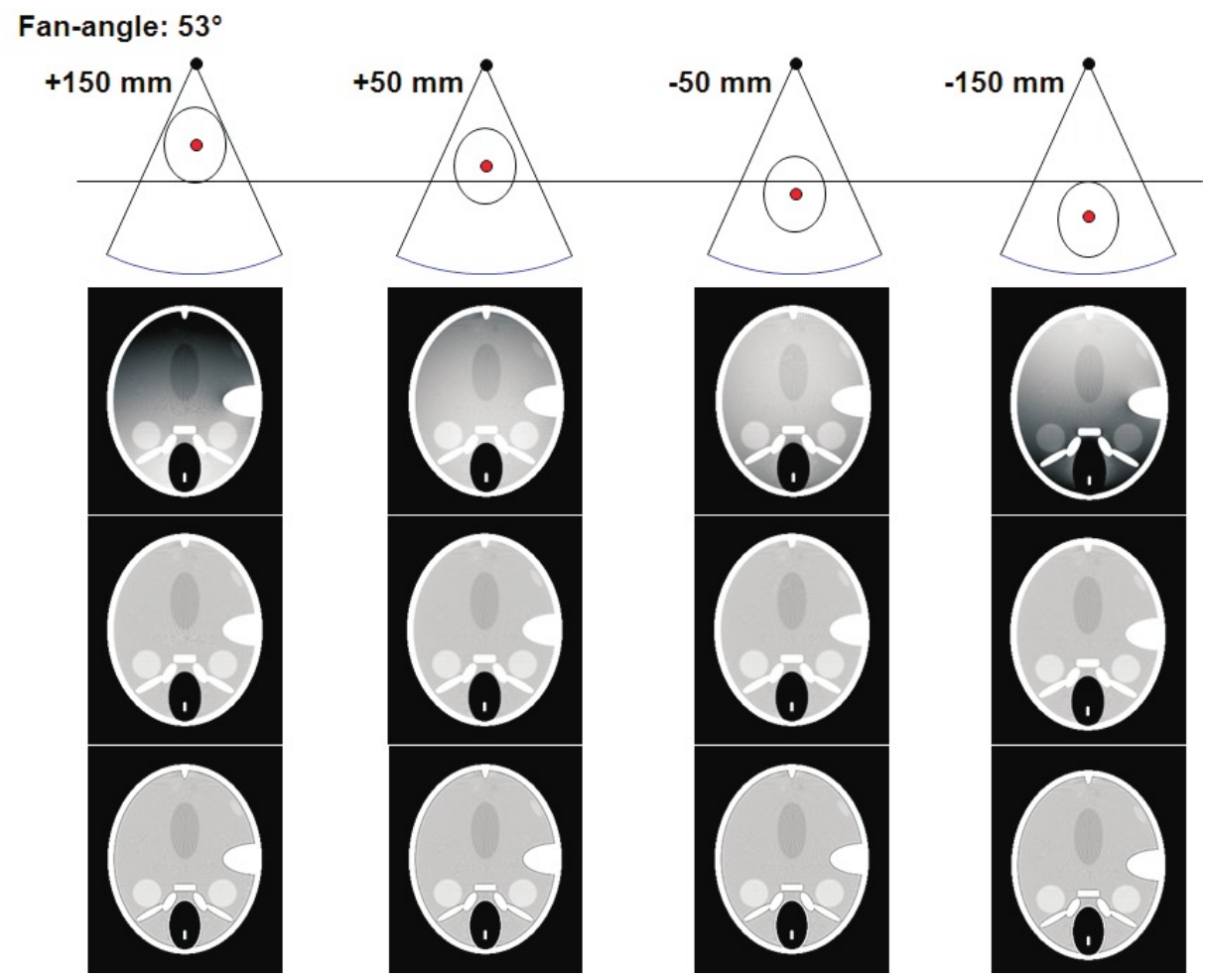

Figure 12. Effect of object-position dependence of visibility loss for various object positions. The upper and middle row of the reconstructed images show the results of a standard FBP reconstruction for the dark-field and attenuation images, respectively. The lower row shows the result of SART for the dark-field images. Level (35 HU) and window (70 HU) are the same for all images.

artifacts in the image. The effect is stronger for larger fan angles (Fig. 11) and for larger off-center positioning of the object (Fig. 12). The observed object-position dependence of visibility loss in dark-field imaging can be explained by a combination of the following effects: first, as the transverse coherence length of the radiation decreases as the object approaches the grating $G_{0}$, the scattering has less influence on the loss of visibility. In the limiting case where the scattering object is right in front of $\mathrm{G}_{0}$, no loss of visibility is seen, as typically the x-ray radiation tube sources can be considered as totally incoherent sources. This is in agreement with the extrapolation from our measurement data. Second, any distance between the scattering object and the grating $\mathrm{G}_{1}$ causes free space propagation of the x-ray wave field leading to a spreading of the scattered radiation, hence dispersing the effect of scattering onto the visibility of the interference fringes. Finally, due to the Fresnel scaling theorem ${ }^{13}$ relating the diffraction patterns of parallel beam geometry to the one of a radial beam geometry (as in our case), any diffraction pattern in the latter geometry gets scaled in intensity and stretched in position as the distance to the observation point $\left(\mathrm{G}_{1}\right)$ in our case increases. A detailed experimental and analytical investigation of how these effects work together is work in progress and shall be published elsewhere.

\section{SUMMARY}

Dark-field imaging overcomes limitations in, for example, CT investigating relatively weakly absorbing material. However, using fan-angle apertures and field of views as those in human CT scanners leads to capping artifacts in the reconstructed images. In this contribution, this scenario is considered and a dedicated reconstruction algorithm is developed taking into account the effect that two complementary x-rays are scattered in a different manner depending on the object position and the size of the scattering features within the object. The application of the described reconstruction algorithm eliminates such artifacts and may be used for dark-field imaging in human CT. 


\section{REFERENCES}

1. F. Pfeiffer, T. Weitkamp, O. Bunk, and C. David, "Phase retrieval and differential phase-contrast imaging with low-brilliance X-ray sources," Nature Phys. 2, pp. 258 - 261, 2006.

2. F. Pfeiffer, M. Bech, O. Bunk, P. Kraft, E. F. Eikenberry, C. Brönnimann, C. Grünzweig, and C. David, "Hard-X-ray dark-field imaging using a grating interferometer," Nature Materials 7, pp. 134 - 137, 2008.

3. M. Bech, O. Bunk, T. Donath, R. Feidenhans'l, C. David, and F. Pfeiffer, "Quantitative X-ray dark-field computed tomography," Phys. Med. Biol. 55, pp. 5529-5539, Sep 2010.

4. S. Schleede, F. Meinel, M. Bech, J. Herzen, K. Achterhold, G. Potdevin, A. Malecki, S. Adam-Neumair, S. Thieme, F. Bamberg, K. Nikolaou, A. Bohla, A. Yildirim, R. Loewen, M. Fifford, R. Ruth, O. Eickelberg, M. Reiser, and F. Pfeiffer, "Emphysema diagnosis using x-ray dark-field imaging at a laser-driven compact synchrotron light source," Proc. Natl. Acad. Sci. USA 109(44), pp. 17880-17885, 2012.

5. W. Yashiro, Y. Terui, K. Kawabata, and A. Momose, "On the origin of visibility contrast in x-ray Talbot interferometry," Opt. Express 18, pp. 16890-16901, Aug 2010.

6. W. Yashiro, S. Harasse, K. Kawabata, H. Kuwabara, T. Yamazaki, and A. Momose, "Distribution of unresolvable anisotropic microstructures revealed in visibility-contrast images using x-ray Talbot interferometry," Physical Review B 84, pp. 094106-1-094106-9, 2011.

7. T. H. Jensen, M. Bech, O. Bunk, T. Donath, C. David, R. Feidenhans'l, and F. Pfeiffer, "Directional x-ray dark-field imaging," Phys. Med. Biol. 55(12), p. 3317, 2010.

8. V. Revol, X-ray Phase Contrast Imaging by Grating Interferometry, PhD Thesis, 2011.

9. Z. T. Wang, K. J. Kang, Z. F. Huang, and Z. Q. Chen, "Quantitative grating-based x-ray dark-field computed tomography," Appl. Phys. Lett. 95(094105), 2009.

10. N. Bevins, J. Zambelli, Z. Qi, and G. Chen, "X-ray dark-field computed tomography using a grating interferometer setup," Medical Imaging 2010: Physics of Medical Imaging 7622(24B), pp. 76220P-76220P6, SPIE, 2010.

11. T. Weitkamp, A. Diaz, C. David, F. Pfeiffer, M. Stampanoni, P. Cloetens, and E. Ziegler, "X-ray phase imaging with a grating interferometer," Opt. Express 13, pp. 6296-6304, Aug 2005.

12. J. R. Taylor, An Introduction to Error Analysis: The Study of Uncertainties in Physical Measurements, University Science Books, 1996.

13. D. M. Paganin, Coherent X-Ray Optics, Oxford University Press, 2006. 